\title{
A CLUSTERING-BASED JUDGMENT METHOD OF FALSE POSITIVE ALERTS
}

\author{
Shinya Iwasaki ${ }^{1}$, Tomo Kakuta ${ }^{1}$, Yoshihiro Sekiguchi ${ }^{1}$, Yukihiro Konishi ${ }^{1}$, Tomoya Ohtori ${ }^{1}$ \\ and Norihisa Komoda ${ }^{2}$ \\ ${ }^{1}$ Hitachi Systems, Ltd., 1-2-1, Osaki, Shinagawa-ku, Tokyo 141-8672, Japan \\ ${ }^{2}$ Code Solutions Co., Ltd., 1-2-11, Edobori, Nishi-ku, Osaka 550-0002, Japan
}

\begin{abstract}
This paper proposes a clustering-based judgment method for false positive alerts generated by security devices. In the proposed method, firstly, alerts with the same attack source IP address over a certain period of time in the past are extracted. After the extraction, using the accumulated signature amount from extracted alerts, the alerts with similar patterns are classified into several clusters using a method combining DBSCAN and K-means++. Then, the judgment of false positive alerts is done based on the number of alerts in each cluster. From a trial usage of the proposed method on two 7-day alert sets from two different networks, it was found that the recall rate was $100 \%$, the precision rate was $34 \%$, and the F-measure was $50 \%$. The precision rate was improved by more than ten times compared to K-means++ alone, and about 2.4 times compared to DBSCAN alone.
\end{abstract}

\section{KEYWORDS}

Cyber Security, Incident Response, Clustering, DBSCAN, K-means++, Anomaly Detection

\section{INTRODUCTION}

In recent years, since the cyber security of companies and public organizations has become increasingly important, it has become necessary to speed up responses to incidents. Here, an incident refers to "a security problem in the operation of the information system." Examples include information leakage, unauthorized access, and falsification of websites. The first action in incident response is to investigate the alerts generated by security devices such as IDS (Intrusion Detection System) and UTM (Unified Threat Management). However, the majority of the massive amount of generated alerts are erroneously detected (Mukherjee, Heberlein and Levitt, 1994). Therefore, since a massive amount of effort is wasted on false positive alerts, these false positive alerts hinder the incident response by cyber security analysts. For this reason, it is necessary to judge and to eliminate these false positive alerts. There are two approaches to judge false positive alerts: use information of alerts only, and use logs of applications layer other than alerts(Munoz, Mana and Javier, 2013). However, since the latter approach requires another sensor and is difficult to implement, the former approach is mainstream. This paper aims to improve the judgment accuracy of false positive alerts by the former approach.

This paper proposes a clustering-based judgment method for false positive alerts. In the proposed method, firstly, alerts with the same attack source IP address over a certain period in the past are extracted. After the extraction, using the accumulated signature amount from extracted alerts, the alerts with similar patterns are classified into several clusters using a method combining DBSCAN (Density-based spatial clustering of applications with noise) (Ester et al., 1996) and K-means++ (Arthur and Vassilvitskii, 2007). Then, the judgment of false positive alerts is done based on the number of alerts in each cluster. 


\section{A JUDGMENT METHOD OF FALSE POSITIVE ALERTS}

Security devices generate security alerts when they detect events that may be attacks. Figure 1 shows an example of an alert generated by a security device. The alert contains a detected time, an attack source IP address, a used communication protocol, and a signature. The signature indicates what type of attack it may have been. This example shows an alert generated by a UTM manufactured by Palo Alto Networks, Inc. This alert indicates that the attacker (source IP address 203.0.113.3) attacked with an attempt at malware infection on June 29, 2017. Alerts occur when the UTM has a pattern of attack traffic defined, called "signature rules." Basic signature rules are defined by UTM vendors. The security administrators can add, delete, and modify the signature rules.

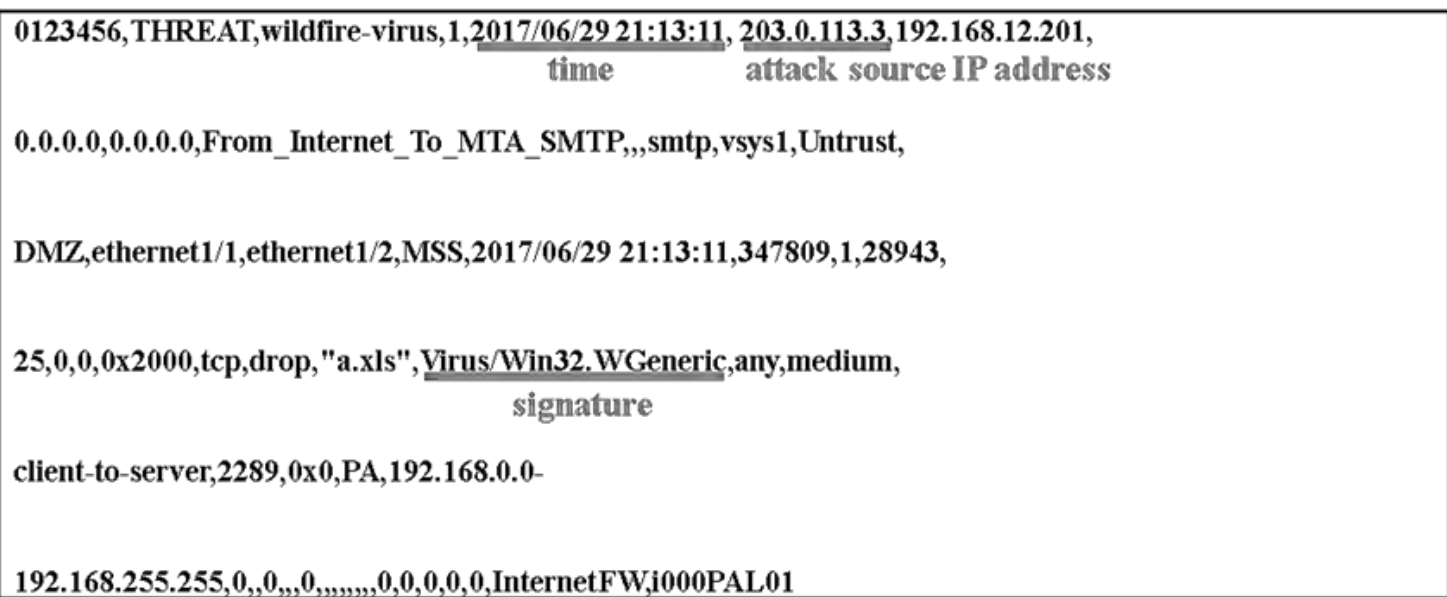

Figure 1. An example of an alert from a security device

The majority of alerts generated by the security devices are not attacks. These alerts are generated because the security device erroneously assesses normal traffic as attack traffic. In this paper, this alert is called an "false positive alert." Normally, the cyber security analysts judge and remove the false positive alerts. However, this operation requires a massive amount of time. Therefore, to shorten the time required for incident response including this, it is necessary to reduce the false positive alerts judged by the cyber security analysts.

Traditional approaches for reducing the time required to judge the false positive alerts are classified into the following three types:

(1) Adjustment of the signature rules of the security devices

The generation of false positive alerts often depends on the characteristics of the network monitored by the security devices. Therefore, the signature rules of the security devices may be added, deleted, and/or modified to match the network characteristics by the security administrator. By adjusting the signature rules, the occurrence of false positive alerts by UTM can be reduced (Juniper Networks, 2016). For example, when the false positive alerts of a specific signature occur frequently, the signature rule generating the false positive alerts should be invalidated. Fujita proposed a new description method for increasing the flexibility of the signature rules (Fujita, 2005). Using this, since the improper rules can be easily modified, the number of false positive alerts may be reduced, and the total time required for the judgment can be shortened. However, since the attack types and vulnerabilities frequently change, this approach is difficult to operate because signature rules must be fine-tuned frequently. On the other hand, outsourced security services are becoming popular in companies and public organizations. Such services often use unified signature rules, because it is very costly to change the signatures for each network. 
(2) Visualization of the alerts

Alerts generated by security devices are difficult for security analysts to understand intuitively. Therefore, methods of visualizing alerts to make them easier to understand for security analysts are proposed. Abdullah reported a study to visualize the occurrence flow of the alerts on a two-dimensional map in association with the local network IP address (Abdullah et al., 2005). Inoue reported on visualization of the communication between the monitoring sensor and the dark net and visualization of the propagation of malware (Inoue et al., 2012). Livnat reported a study on visualization of the correlation by grouping alerts (Livnat et al., 2005). Their studies are aimed at reducing the time required for the false positive alert judgment by security analysts. However, it is difficult for security analysts to judge all of the generated alerts. Therefore, this method alone is insufficient to shorten the time required for the false positive alert judgments.

(3) Judgment alerts by using a mechanical method

A typical mechanical method is the "unsupervised anomaly detection method" (Eskin et al., 2002). In the security field, studies using an unsupervised anomaly detection method are popular because the tendencies of attacks change in a relatively short period of time due to the growing sophistication of attacks, discovery of vulnerabilities, changes in trends, and the like (Portnoy, Eleazar and Stolfo, 2001) (Muda et al., 2011) (Depren et al., 2005) (Liang Hu et al., 2015). The unsupervised anomaly detection method is a method of classifying alerts with similar tendencies. Because the set of the alerts consists of many false positive alerts and a few true attack alerts, the false positive alerts and the attack alerts can be classified by the unsupervised anomaly detection method. This method can classify input alerts only, and it is not necessary to study in advance with teacher data. However, the unsupervised detection method is inferior in accuracy to other anomaly detection methods such as the supervised abnormality detection method. Yin proposed a judgment method using RNN and reported that it is more accurate than the existing judgment method using random forest and support vector machine(Yin et al., 2017). However, to use the supervised detection method in the security field, it is necessary to regularly learn and update the judgment method.

\section{A CLUSTERING-BASED JUDGMENT METHOD}

\subsection{Process of the Proposed Judgment Method}

Since recent cyber-attacks are often the combination of the multiple attack methods, this type of attack causes alerts with various signatures in a single attack. On the other hand, the false positive alerts are characterized by a large amount of alerts with specific signatures. Therefore, the alerts that detect attacks correctly are considered to have different tendencies in signatures compared to the false positive alerts. Considering this characteristic, it is possible to classify the alerts into several groups by using the clustering method based on the signatures in the alerts. The alerts in the large groups are judged as the false positive alerts, because the false positive alerts consist of alerts with many similar signatures. By using clustering, the judgment of the false positive alerts can be done only from alert information without depending on the network.

The proposed method is composed of the following three steps:

(1) Extraction of alerts with the same attacker IP address

For each alert generated by the security device, the alerts having the same attacker IP address are extracted from the alerts over a certain period of time in the past.

(2) Calculation of generated alerts with same signature name from the extracted alerts.

Section 4.2 explains this step in detail.

(3) Judgment of false positive alerts by using clustering.

Section 4.3 explains this step in detail. 


\subsection{Calculation of Generated Alerts}

In this step, signature trends are extracted from alerts for cluster classification.

Firstly, the generated amount by the signature name is aggregated from the alerts extracted in the previous step. For example, in Figure 2, let us use "Alert 4" as an evaluated alert. "Alert 1" and "Alert 2," which have the same attacker IP address (203.0.113.3) as "Alert 4" within a certain period in the past, are extracted. By aggregating the signature names of the extracted "Alert 1 " and "Alert 2, " the signature $\mathrm{A}$ is one time and the signature B is two times. By executing this operation for all alerts, the generation amount by signature name is aggregated.

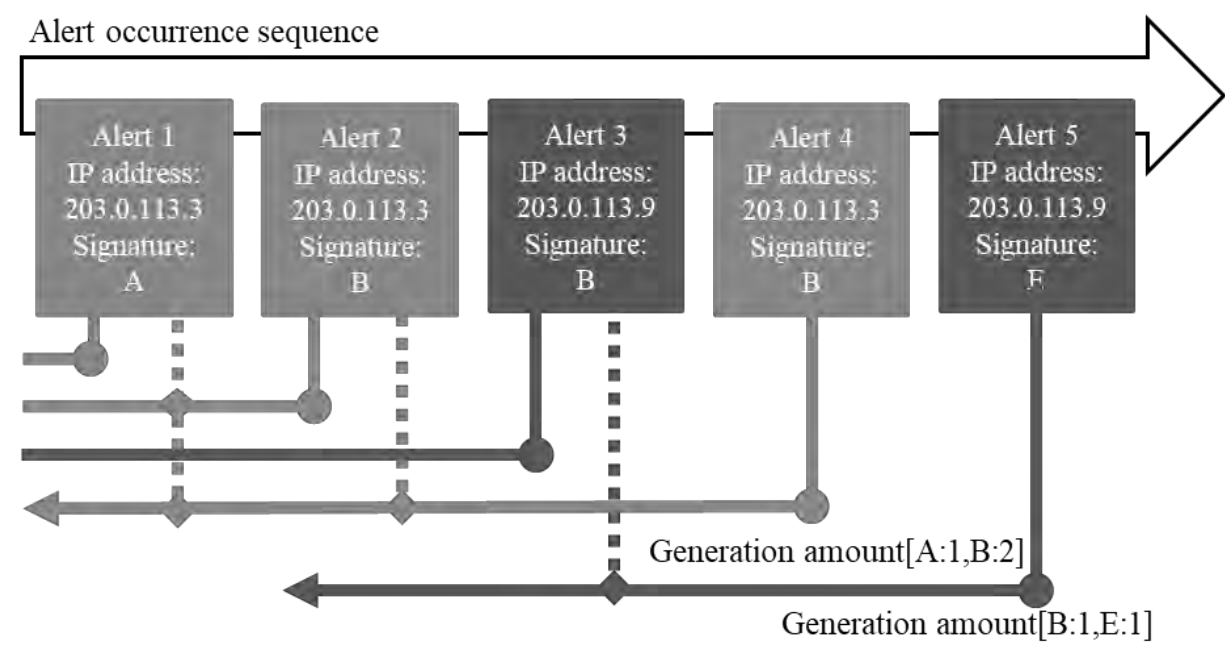

Figure 2. Aggregation of generated amount by signature in alert

Next, the result of counting all alerts is converted to a matrix form. A signature which has not occurred is filled with 0 . For example, in the case of the previous example, the result of aggregating "alerts 1-5" is Table 1.

Table 1. Matrix example of aggregated result

\begin{tabular}{|c|c|c|c|}
\hline & Signature A & Signature B & Signature E \\
\hline Alert 1 & 1 & 0 & 0 \\
\hline Alert 2 & 1 & 1 & 0 \\
\hline Alert 3 & 0 & 1 & 0 \\
\hline Alert 4 & 1 & 2 & 0 \\
\hline Alert 5 & 0 & 1 & 1 \\
\hline
\end{tabular}

The generation amount by signature name of one alert is called a "signature pattern." The signature pattern represents the trend of the event that generated the targeted alert.

\subsection{Judgment by Clustering}

If the learning models such as SVM (support vector machine) are used for classification, before learning, it is necessary to preliminarily assign the false positive alert flag to the alerts. On the other hand, in the clustering method, as shown in the right side of Figure 3, the classification can be done only from the alert information. Since there is no need to assign a flag, the clustering method is suitable for the judgment of the false positive alerts. 
In the proposed method, the alerts are classified together into several clusters having the same pattern by using clustering based on aggregation results. The clustering methods DBSCAN and K-means++ are combined. By combining the clustering methods, the false positive alerts are judged as much as possible while correctly judging the attack alerts.

Figure 4 shows the flow of judgment of alerts. Most false positive alerts of similar signature patterns are judged by classification of DBSCAN. After that, false positive alerts among the remaining alerts are judged by classification using K-means++. DBSCAN is an algorithm that constitutes a cluster according to the density of input data. In this method, mechanically optimal clusters are determined with the distance threshold (eps) and the minimum inclusion count (minPts). For this reason, since DBSCAN can classify outliers not belonging to any cluster, DBSCAN can separately classify the alerts into large clusters of the false positive alerts and the isolated attack alerts from the clusters.

Therefore, at first, based on the aggregation result in the previous step, the alerts are classified by DBSCAN. As a result of classification by DBSCAN, the alerts belonging to the cluster are judged as "false positive alerts." Then, the alerts belonging to outliers are classified by K-means++. In the K-means++ classification, input data can be classified into clusters of a specified number. As a result, the alerts belonging to outliers can be classified as false positive alerts or attack alerts.
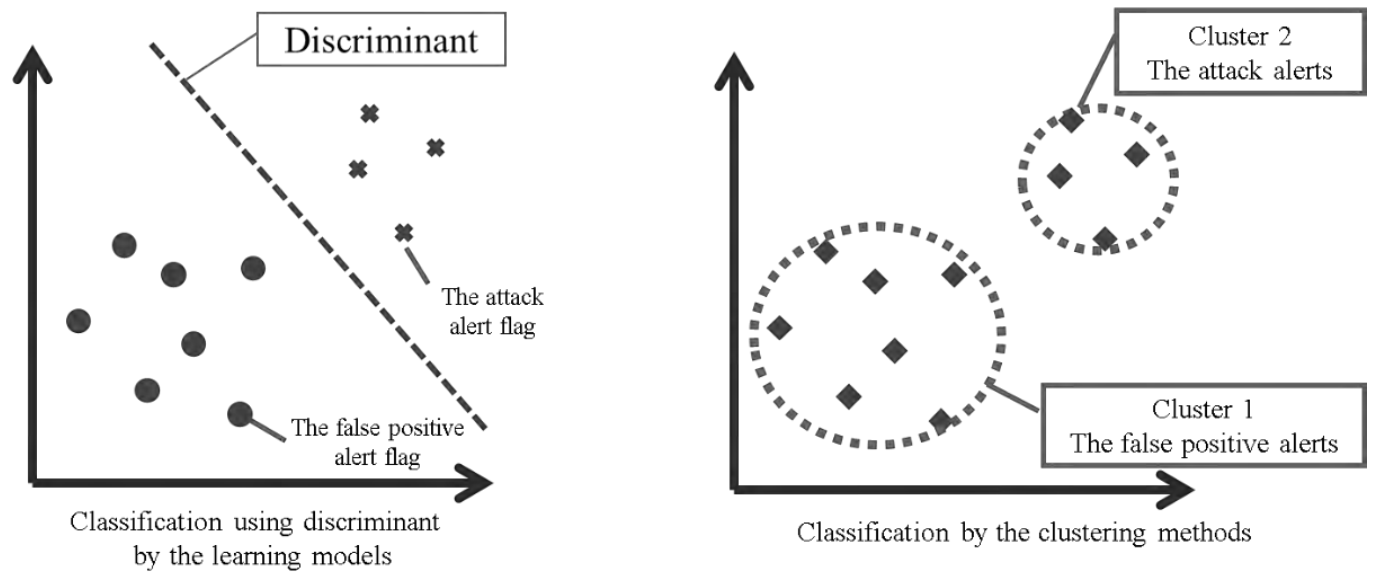

Figure 3. A model of the difference between learning models and clustering methods

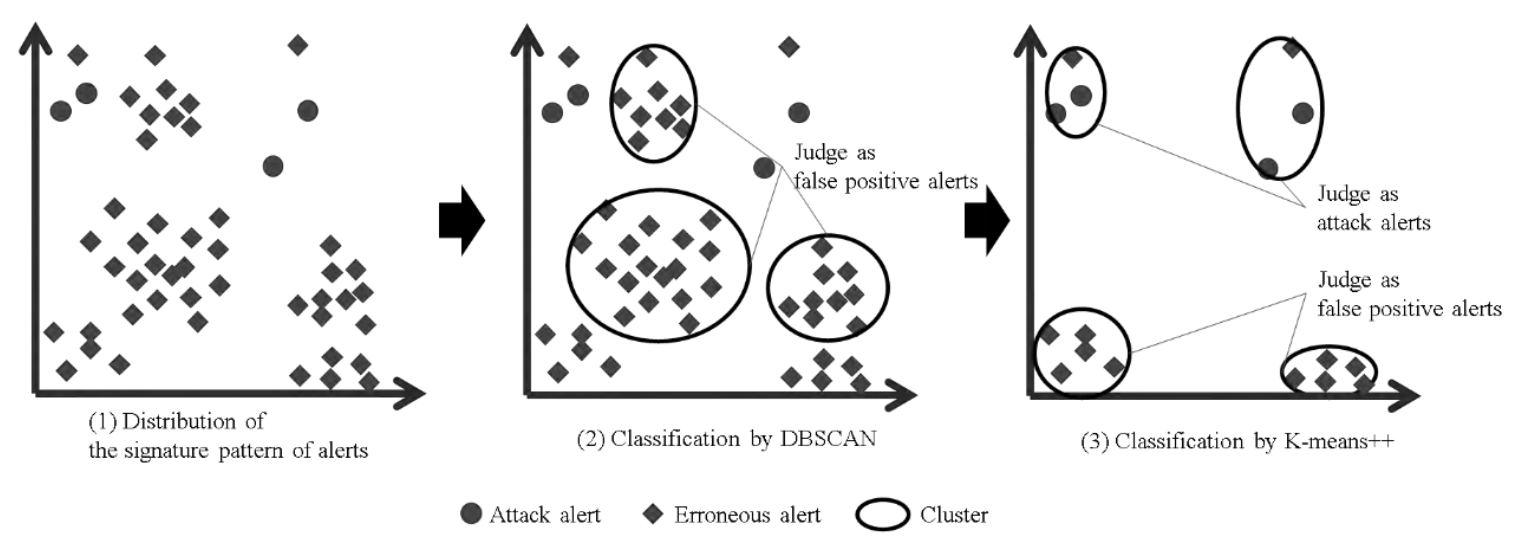

Figure 4. Flow of judgment of alerts using clustering by the proposed method 


\section{EXPERIMENT}

The proposed method was applied to two data sets. Table 2 shows the characteristics of the alert set used for evaluation. Datasets $\mathrm{A}$ and $\mathrm{B}$ are 7 days of alerts from different security devices monitoring different networks. "Attack alert" and "False positive alert" are judged by cyber security analysts. For comparison, in addition to the proposed method, K-means++ only and DBSCAN only were also evaluated. The "certain period of time in the past" was 30 minutes based on the opinion of cyber security analysts.

The result is shown in Table 3 and 4. As a result of classification by DBSCAN, Dataset A was classified into 10 clusters, and Dataset B was classified into 3 clusters.

Table 2. Data set and judgment result by the cyber security analysts

\begin{tabular}{|c|c|c|}
\hline Dataset & Attack alerts & False positive alerts \\
\hline A & 7 & 7023 \\
\hline B & 9 & 13223 \\
\hline
\end{tabular}

Table 3. The evaluation result by three methods

\begin{tabular}{|c|c|c|c|c|c|}
\hline \multicolumn{2}{|c|}{} & \multicolumn{2}{c|}{ Dataset A } & \multicolumn{2}{c|}{ Dataset B } \\
\cline { 3 - 6 } & Attack alerts & 7 & 364 & 9 & 174 \\
\hline \multirow{2}{*}{ K-means++ alone } & Attack alerts & $\begin{array}{c}\text { False positive } \\
\text { alerts }\end{array}$ & Attack alerts & $\begin{array}{c}\text { False positive } \\
\text { alerts }\end{array}$ \\
\cline { 2 - 6 } & $\begin{array}{c}\text { False positive } \\
\text { alerts }\end{array}$ & 0 & 6659 & 0 & 13049 \\
\hline \multirow{2}{*}{$\begin{array}{c}\text { DBSCAN alone } \\
\text { Attack alerts }\end{array}$} & $\begin{array}{c}\text { False positive } \\
\text { alerts }\end{array}$ & 0 & 96 & 9 & 32 \\
\hline $\begin{array}{c}\text { Proposed method } \\
\text { (DBSCAN }\end{array}$ & Attack alerts & 7 & 22 & 0 & 13191 \\
\cline { 2 - 6 } & $\begin{array}{c}\text { False positive } \\
\text { alerts }\end{array}$ & 0 & 7001 & 9 & 11 \\
\hline
\end{tabular}

Table 4. The accuracy of three methods

\begin{tabular}{|c|c|c|c|}
\hline & Recall rate & Precision rate & F-measure \\
\hline K-means++ alone & 1.00 & 0.03 & 0.14 \\
\hline DBSCAN alone & 1.00 & 0.14 & 0.24 \\
\hline $\begin{array}{c}\text { Proposed method } \\
\text { (DBSCAN \& K-means++) }\end{array}$ & 1.00 & 0.34 & 0.50 \\
\hline
\end{tabular}

16 attack alerts in total of datasets A and B were properly judged as attacks by all methods, and the recall rate for attacks was $100 \%$. The $100 \%$ recall rate is important for cyber security because attacks cannot be overlooked. However, there are differences in false positives in 538 cases for "K-means++ only," 128 cases for "DBSCAN only," and 33 cases for the proposed method. As a result, the precision rate of the proposed method is $34 \%$ and the F-measure is $50 \%$, which is the best when compared to other methods. The reason is that as a result of classification by DBSCAN, attack alerts of unique signature patterns were classified as outliers. Furthermore, by classifying the alerts of outliers using K-means++, it is possible to appropriately judge false positive alerts rarely occurring. 


\section{CONCLUSION}

This paper proposed a clustering-based judgment method for false positive alerts generated by security devices. In the proposed method, firstly, alerts with the same attack source IP address over a period of time in the past are extracted. After the extraction, using the accumulated signature amount from extracted alerts, alerts with similar patterns are classified into several clusters using a method combining DBSCAN and K-means++. Then, the judgment of false positive alerts is done based on the number of alerts in each cluster. From a trial usage of the proposed method on two 7-day alert sets from two different networks, it was found that the recall rate was $100 \%$, the precision rate was $34 \%$, and the F-measure was $50 \%$. The precision rate was improved by more than ten times compared to K-means++ alone, and about by 2.4 times compared to DBSCAN alone. The reason is that as a result of classification by DBSCAN, attack alerts of unique signature patterns were classified as outliers. Furthermore, by classifying the alerts of outliers using K-means++, it is possible to appropriately judge false positive alerts rarely occurring.

\section{REFERENCES}

Abdullah, K. et al., 2005. IDS RainStorm: Visualizing IDS Alarms. Proceedings of IEEE Workshop on Visualization for Computer Security. pp. 1-10.

Arthur, D. and Vassilvitskii, S., 2007. k-means++: the advantages of careful seeding. Proceedings of the eighteenth annual ACM-SIAM symposium on Discrete algorithms. pp. 1027-1035.

Depren, O. et al., 2005. An Intelligent intrusion detection system (IDS) for anomaly and misuse detection in computer networks. Expert Systems with Applications. 29(4), pp. 713-722.

Eskin, E. et al., 2002. A Geometric Framework for Unsupervised Anomaly Detection. Proceedings of Applications of Data Mining in Computer Security. Springer, Boston, pp. 77-101.

Ester, M. et al., 1996. A density-based algorithm for discovering clusters in large spatial databases with noise. Proceedings of Second International Conference on Knowledge Discovery and Data Mining. pp. 226-231.

Fujita, N., 2005. Development of Injustice Access Processing System That Reduces Log Output Using a Flexible Description for the Intrusion Detection Policy. The transactions of the Institute of Electronics, Information and Communication Engineers, 88(2), pp. 391-400 (in Japanese).

Inoue, D. et al., 2008. nicter: An Incident Analysis System Toward Binding Network Monitoring with Malware Analysis. Proceedings of WOMBAT Workshop on Information Security Threats Data Collection and Sharing. pp. 58-66.

Juniper Networks, 2016. Understanding IPS Signatures - TechLibrary. Juniper. Available at: https://www.juniper.net/documentation/en_US/junos-space15.2/topics/concept/junos-space-ips-policy-signatureunderstanding.html (Accessed: 31 March 2019).

Liang Hu et al., 2015. False positive elimination in intrusion detection based on clustering. 12th International Conference on Fuzzy Systems and Knowledge Discovery (FSKD). pp. 519-523.

Livnat, Y. et al., 2005. A visualization paradigm for network intrusion detection. Proceedings of the Sixth Annual IEEE Systems, Man and Cybernetics (SMC) Information Assurance Workshop. pp. 92-99.

Muda, Z. et al., 2011. Intrusion Detection based on K-Means Clustering and Naïve Bayes Classification. Proceedings of the 7th International Conference on IT in Asia (CITA'11). pp. 1-6.

Mukherjee, B., Heberlein, L. T. and Levitt, K. N., 1994. Network intrusion detection. IEEE Network. 8(3), pp. $26-41$.

Munoz, A., Mana, A. and Javier, G., 2013. Dynamic Security Properties Monitoring Architecture for Cloud Computing, Security Engineering for Cloud Computing: Approaches and Tools. IGI Publishing Hershey, pp. 1-18.

Portnoy, L., Eleazar, E. and Stolfo, S., 2001. Intrusion detection with unlabeled data using clustering. Proceedings of ACM CSS Workshop on Data Mining Applied to Security. pp. 5-8.

Yin, C. et al., 2017. A Deep Learning Approach for Intrusion Detection Using Recurrent Neural Networks. IEEE Access. 5, pp. 21954-21961. 